\title{
A Phase I study of pazopanib in combination with gemcitabine in patients with advanced solid tumors
}

\author{
Ruth Plummer • Ayman Madi $\cdot$ Melinda Jeffels $\cdot$ Heike Richly $\cdot$ Bahar Nokay • \\ Stephen Rubin - Howard A. Ball · Steve Weller · Jeffrey Botbyl • \\ Diana M. Gibson • Max E. Scheulen
}

Received: 18 July 2012 / Accepted: 15 September 2012/Published online: 11 October 2012

(C) The Author(s) 2012. This article is published with open access at Springerlink.com

\begin{abstract}
Purpose Pazopanib plus gemcitabine combination therapy was explored in patients with advanced solid tumors. Methods In a modified $3+3$ enrollment scheme, oral once-daily pazopanib was administered with intravenous gemcitabine (Days 1 and 8, 21-day cycles). Three protocolspecified dose levels were tested: pazopanib $400 \mathrm{mg}$ plus gemcitabine $1,000 \mathrm{mg} / \mathrm{m}^{2}$, pazopanib $800 \mathrm{mg}$ plus gemcitabine $1,000 \mathrm{mg} / \mathrm{m}^{2}$, and pazopanib $800 \mathrm{mg}$ plus gemcitabine $1,250 \mathrm{mg} / \mathrm{m}^{2}$. Maximum-tolerated dose was based on dose-limiting toxicities during treatment Cycle 1. In the expansion phase, six additional patients were enrolled at the highest tolerable dose level.

Results Twenty-two patients were enrolled. At the highest dose level tested (pazopanib 800 plus gemcitabine
\end{abstract}

R. Plummer $(\square) \cdot$ A. Madi · M. Jeffels

Sir Bobby Robson Cancer Trials Research Centre, Northern

Centre for Cancer Care, Newcastle upon Tyne NE7 7DN, UK

e-mail: ruth.plummer@newcastle.ac.uk;

Ruth.plummer@ncl.ac.uk

H. Richly · B. Nokay · M. E. Scheulen

Department of Medical Oncology, West German Cancer Center,

University Hospital Essen, University of Duisburg-Essen,

Essen, Germany

S. Rubin · H. A. Ball

GlaxoSmithKline, Collegeville, PA, USA

Present Address:

H. A. Ball

Astellas, Deerfield, IL, USA

S. Weller - D. M. Gibson

GlaxoSmithKline, Research Triangle Park, NC, USA

J. Botbyl

Provonix, Mullica Hill, NJ, USA
$1,250)$, patients received $>80 \%$ of their planned dose and the regimen was deemed safe and tolerable. The most common treatment-related adverse events included fatigue, neutropenia, nausea, and decreased appetite. Neutropenia and thrombocytopenia were the most common events leading to dose modifications. Pharmacokinetic interaction between pazopanib and gemcitabine was not observed. One objective partial response at the highest dose was observed in a patient with metastatic melanoma. Prolonged disease stabilization ( $>12$ cycles) was reported in three patients (metastatic melanoma, cholangiocarcinoma, and colorectal carcinoma).

Conclusion Combination pazopanib plus gemcitabine therapy is tolerable, with an adverse event profile reflective of that associated with the individual agents. There was no apparent pharmacokinetic interaction with pazopanib plus gemcitabine co-administration, although patient numbers were limited. Further investigation of combined pazopanib plus gemcitabine is warranted.

Keywords Anti-angiogenesis - Combination therapy · Gemcitabine · Melanoma · Pazopanib - Pharmacokinetics · Phase I · Solid tumors

\section{Introduction}

Pazopanib is a multi-tyrosine kinase inhibitor of vascular endothelial growth factor receptor (VEGFR)-1, VEGFR-2, VEGFR-3, platelet-derived growth factor receptor (PDGFR)- $\alpha$, PDGFR- $\beta$, fibroblast growth factor receptor (FGFR)-1, FGFR-3, and c-Kit. Pazopanib is approved as monotherapy for patients with advanced renal cell carcinoma [1] and soft tissue sarcoma [2] and is currently under investigation in multiple tumor types, including ovarian 
cancer, non-small-cell lung cancer, thyroid cancer, and cervical cancer [3-7].

Gemcitabine is a cytotoxic nucleoside analogue of deoxycytidine whose triphosphate ( $\mathrm{dFdCTP})$ is irreversibly incorporated into DNA, subsequently inhibiting exonuclease and DNA repair activities. Gemcitabine has broadspectrum activity and is approved or commonly used, either as a single agent or in combination with other chemotherapy agents, for the treatment of ovarian cancer, breast cancer, non-small-cell lung cancer, pancreatic cancer, and soft tissue sarcoma [8-12]. Myelosuppression was the dose-limiting toxicity (DLT) in gemcitabine singleagent Phase I trials [13, 14].

Clinical studies exploring therapeutic strategies that combine angiogenesis pathway inhibition with concurrent chemotherapy have shown promise for the treatment of various malignancies [15-17]. Therefore, a Phase I study (NCT00678977; VEG109599) was conducted to determine the maximum-tolerated dose (MTD) of pazopanib in combination with gemcitabine. Secondary objectives included evaluation of safety and pharmacokinetics of the combination and assessment of the preliminary clinical activity in patients with advanced solid tumors.

\section{Patients and methods}

\section{Study participants}

Eligible patients were at least 18 years of age with a histologically or cytologically confirmed advanced solid tumor, who had progressed on standard therapy or for whom no standard therapy was available. Additional eligibility criteria included an Eastern Cooperative Oncology Group (ECOG) performance status of 0 or 1; measurable or evaluable disease at the time of screening; adequate hematologic, hepatic, and renal function; and no unstable or serious concurrent medical condition. An unlimited number of prior therapies were permitted; however, at least 4 weeks must have elapsed since previous treatment. Patients with asymptomatic brain metastases who did not require steroids and antiseizure medications for more than 3 months were eligible.

Exclusion criteria included the presence of leptomeningeal carcinomatosis; clinically significant gastrointestinal abnormality; elevated blood pressure $(\geq 140 / 90 \mathrm{mmHg})$; prolonged QT interval $(>480 \mathrm{~ms})$; history of cardiac angioplasty or stenting, myocardial infarction, unstable angina, symptomatic peripheral vascular disease, or Class III or IV congestive heart failure; uncontrolled infection; history of cerebrovascular accident, pulmonary embolism, or untreated deep vein thrombosis within the previous 6 months; and previous treatment with an investigational or licensed tyrosine kinase inhibitor targeting VEGF receptors.

The study was conducted in accordance with the standards of each site's independent ethics committees, principles of good clinical practice, all applicable regulatory requirements, and the Declaration of Helsinki. All patients provided written informed consent before enrollment and before undergoing any study-specific procedures.

\section{Study design}

This open-label study consisted of a dose-escalation phase to determine the MTD and a fixed-dose, cohort-expansion phase to further define the safety and tolerability of the MTD. The dose-escalation phase used a $3+3$ enrollment design. Initially, three patients were enrolled into Dose Level 1; if no DLT was observed, three patients were enrolled at the next dose level. If a DLT was observed in one of the first three patients enrolled at a given dose level, three additional patients were enrolled at that dose level. Escalation to the subsequent dose level was permitted if no more than one of six patients experienced a DLT. If, however, two or more patients experienced a DLT at a given dose level, the MTD was considered to have been exceeded and a lower or intermediate dose level would be explored.

Dose-limiting toxicity was based on observed toxicity during the first cycle of treatment. Toxicities included the following: Grade 4 neutropenia, febrile neutropenia, Grade 4 anemia, platelet count below 25,000 , serum creatinine at least 2 times baseline or upper limit of normal, Grade 3 proteinuria with uncontrolled hypertension or renal impairment and Grade 4 proteinuria, Grade 3 or higher non-hematologic toxicity (except fatigue but including nausea, vomiting, and diarrhea not controlled by supportive treatment), Grade 3 uncontrolled hypertension, Grade 4 hypertension, delay of treatment for more than 3 weeks, or inability to receive $75 \%$ of scheduled doses in a treatment cycle.

\section{Treatment}

During the dose-escalation phase, 3 protocol-defined dose regimens were evaluated. In this phase, pazopanib was administered orally once daily beginning on Day 1 of Cycle 1, and gemcitabine was administered intravenously on Day 1 and Day 8 of each 21-day cycle. In the cohortexpansion phase, gemcitabine was administered on the same schedule, but daily pazopanib dosing began on Day 2 of Cycle 1 to permit determination of gemcitabine-alone pharmacokinetics on Day 1. Gemcitabine infusions were administered over $30 \mathrm{~min}$. Intrapatient dose escalations were not permitted. Dose modifications and reductions for 
pazopanib were to be performed for the control of blood pressure or in the event of hemorrhage, thrombosis, proteinuria, or hepatotoxicity. Dose modifications and reductions for gemcitabine were based on hematologic toxicity and other toxicities causally related to gemcitabine. Treatment could continue in the absence of unacceptable toxicities, disease progression, patient withdrawal of consent, investigator decision, or a delay in treatment for more than 3 weeks.

\section{Patient evaluation}

Screening assessments were completed within 28 days before the first dose of study treatment; these included medical history, prior anticancer therapy, physical examination, ECOG performance status, vital signs, hematology, and clinical chemistry. Baseline electrocardiogram and echocardiogram or multi-gated acquisition (MUGA) scans were also performed.

Safety was assessed throughout the study by physical examination, 12-lead electrocardiograms, echocardiograms or MUGA, vital sign measurements, and clinical laboratory tests. Patients were monitored for adverse events (AEs) throughout the study. The frequency, severity, and relationship to treatment for AEs that occurred during study treatment and up to 30 days after the last dose of study drug were evaluated. Adverse events were coded according to the Medical Dictionary for Regulatory Activities (MedDRA) and Common Terminology Criteria for Adverse Events (CTCAE) version 3.0. All patients who received at least one dose of study drug were included in the safety analyses.

Disease assessment was performed within 28 days before the first dose of study treatment and every 2 treatment cycles thereafter. All patients completing at least 2 treatment cycles were evaluable for response. Tumor response was evaluated according to Response Evaluation Criteria in Solid Tumors [18]. Confirmatory scans were required at least 4 weeks after the initial documentation of a complete or partial response.

\section{Pharmacokinetic sampling}

Sparse sampling was performed during the dose-escalation phase; serial blood samples for plasma pazopanib analysis were nominally collected pre-dose and $3.5 \mathrm{~h}$ post-dose on Day 1 of Cycle 1, and pre-dose on Day 8 of Cycle 1 and Day 1 of Cycle 2. Blood samples for analysis of plasma gemcitabine and its metabolite, $2^{\prime}, 2^{\prime}$-difluorodeoxyuridine (dFdU), were nominally collected pre-dose and at $0.5 \mathrm{~h}$ (i.e., at the end of gemcitabine infusion) on Days 1 and 8 of Cycle 1. In the cohort-expansion phase, blood samples for plasma gemcitabine and $\mathrm{dFdU}$ analysis were collected pre-dose and at $0.25,0.5,1,1.5,2,4,6,8$, and $24 \mathrm{~h}$ after the start of the gemcitabine infusion on Day 1 of Cycle 1 (gemcitabine alone) and at the same times on Day 1 of Cycle 2 (gemcitabine and pazopanib in combination) for gemcitabine, $\mathrm{dFdU}$, and pazopanib analysis.

\section{Drug concentration assays}

Plasma samples were analyzed for pazopanib using a validated analytical method based on protein precipitation, followed by high-performance liquid chromatography/tandem mass spectrometry (HPLC/MS/MS) analysis [19]. The lower limit of quantification (LLQ) was $100 \mathrm{ng} / \mathrm{mL}$, using a $20-\mu \mathrm{L}$ aliquot of human plasma with a higher limit of quantification (HLQ) of $50,000 \mathrm{ng} / \mathrm{mL}$. Plasma concentrations of gemcitabine and $\mathrm{dFdU}$ were determined using a validated method based on liquid/liquid extraction with methyl tertiary-butyl ether (MTBE) and chemical derivatization with dansyl chloride, followed by HPLC/MS/MS analysis. The LLQ for gemcitabine and $\mathrm{dFdU}$ was 50 and $500 \mathrm{ng} / \mathrm{mL}$, respectively, using a $50-\mu \mathrm{L}$ aliquot of human plasma with HLQ of $50,000 \mathrm{ng} / \mathrm{mL}$ for both gemcitabine and dFdU. For each assay, quality control (QC) samples, prepared at three different analyte concentrations and stored with study samples, were analyzed with each batch of samples against separately prepared calibration standards. For the analysis to be acceptable, no more than onethird of the QC results were to deviate from the nominal concentration by more than $15 \%$, and at least $50 \%$ of the results from each QC concentration would be within $15 \%$ of nominal.

\section{Statistical and pharmacokinetic analysis}

Statistical analyses were performed using the SAS/STAT module of SAS, version 9 (SAS Institute, Cary, NC). Pharmacokinetic concentrations (dose-escalation and expansion cohorts) and pharmacokinetic parameters (expansion cohort only) for pazopanib, gemcitabine, and dFdU were summarized by dose cohort. In the expansion cohort, for each of the analytes, pharmacokinetic parameter estimates were obtained for maximum concentration $\left(C_{\max }\right)$, time of $C_{\max }$, area under the plasma concentration-time curve (AUC) from time 0 to time post-dose of last quantifiable concentration, and elimination half-life. For gemcitabine, AUC extrapolated to infinity $\left(\mathrm{AUC}_{(0-\infty)}\right)$ and systemic clearance were estimated; $\mathrm{AUC}_{(0-\infty)}$ was also estimated for dFdU. To assess the potential effect of pazopanib on gemcitabine pharmacokinetics, gemcitabine and $\mathrm{dFdU}$ pharmacokinetic parameters $C_{\max }, \mathrm{AUC}_{(0-\infty)}$, and elimination half-life were $\log _{\mathrm{e}}$-transformed and analyzed by mixed-effect analysis of variance (ANOVA), fitting terms for treatment (test: pazopanib plus gemcitabine [Cycle 2, Day 1]; reference: gemcitabine alone 
[Cycle 1, Day 1]) as a fixed effect and patient as a random effect. Geometric least squares means and $90 \%$ confidence intervals $(\mathrm{CI})$ for the differences in $\log _{\mathrm{e}}$-transformed parameters were then back-transformed to obtain the geometric mean ratio (test/reference) and associated $90 \% \mathrm{CI}$ on the original scale.

Adverse events were listed and summarized by treatment regimen and the percent of patients reporting each event at least once. Laboratory parameters, vital signs, and electrocardiograms were summarized by time point and treatment regimen. Dose intensity was defined as ([actual dose/planned dose] $\times 100)$.

\section{Results}

Patient characteristics

Between April 2008 and March 2010, a total of 22 patients with advanced solid tumors were enrolled and treated with pazopanib plus gemcitabine; 21 patients completed the study. Two patients with melanoma who received pazopanib $800 \mathrm{mg}$ plus gemcitabine $1,250 \mathrm{mg} / \mathrm{m}^{2}$ (Paz800/ Gem1250) continued pazopanib treatment for an additional 14 and 16 months, respectively, after database lock. The most frequent tumor type was melanoma (eight patients, $36 \%$; Table 1). All patients had received at least 1 prior line of chemotherapy; 13 patients $(59 \%)$ had received at least 2 prior lines of chemotherapy.

Dose escalation and determination of MTD

A DLT of Grade 4 thrombocytopenia was reported in one of the initial three patients (Table 2) enrolled in Dose Level 1 (pazopanib $400 \mathrm{mg}$ plus gemcitabine $1,000 \mathrm{mg} / \mathrm{m}^{2}$ [Paz400/Gem1000]). As a result, Dose Level 1 was expanded to a total of six patients. No further DLTs were reported in Dose Level 1. No DLTs were observed in the dose-escalation phase of Dose Level 2 (pazopanib $800 \mathrm{mg}$ plus gemcitabine $1,000 \mathrm{mg} / \mathrm{m}^{2}$ [Paz800/Gem1000]) or in the first three patients enrolled in Dose Level 3 (Paz800/ Gem1250). Because Dose Level 3 was the highest protocoldefined dose level at which pazopanib and gemcitabine were administered at therapeutic levels equivalent to that of monotherapy with the individual agents, additional dose levels were not evaluated. Thus, the MTD was not determined. Overall, 1 DLT (Grade 3 fatigue) was reported among 13 patients enrolled in Dose Level 3 in the doseescalation and cohort-expansion phase.

Table 1 Patient demographics and disease characteristics at baseline

\begin{tabular}{|c|c|c|c|c|}
\hline & Paz400/Gem1000 $(n=6)$ & Paz800/Gem1000 $(n=3)$ & Paz800/Gem1250 $(n=13)$ & Total $(N=22)$ \\
\hline \multicolumn{5}{|l|}{ Gender $(n \%)$} \\
\hline Female & $4(67)$ & $2(67)$ & $5(38)$ & $11(50)$ \\
\hline Male & $2(33)$ & $1(33)$ & $8(62)$ & $11(50)$ \\
\hline \multicolumn{5}{|l|}{ Race $(n \%)$} \\
\hline White & $6(100)$ & $3(100)$ & $13(100)$ & $22(100)$ \\
\hline \multicolumn{5}{|l|}{ Ethnicity $(n \%)$} \\
\hline Hispanic or Latino & 0 & 0 & 0 & 0 \\
\hline Not Hispanic or Latino & $6(100)$ & $3(100)$ & $13(100)$ & $22(100)$ \\
\hline Median age, years (range) & $56(22-63)$ & $49(47-52)$ & $63(30-74)$ & $56(22-74)$ \\
\hline \multicolumn{5}{|l|}{ ECOG PS $(n \%)$} \\
\hline 0 & $2(33)$ & $3(100)$ & $9(69)$ & $14(64)$ \\
\hline 1 & $4(67)$ & 0 & $4(31)$ & $8(36)$ \\
\hline \multicolumn{5}{|l|}{ Primary tumor $(n \%)$} \\
\hline Melanoma & $1(17)$ & $1(33)$ & $6(46)$ & $8(36)$ \\
\hline NSCLC & 0 & 0 & $3(23)$ & $3(14)$ \\
\hline Colorectal & $1(17)$ & $1(33)$ & $1(8)$ & $3(14)$ \\
\hline Cervix & $1(17)$ & 0 & 0 & $1(5)$ \\
\hline Esophagus & 0 & 0 & $1(8)$ & $1(5)$ \\
\hline Ovarian & $1(17)$ & 0 & 0 & $1(5)$ \\
\hline Stomach & 0 & 0 & $1(8)$ & $1(5)$ \\
\hline Other & $2(33)$ & 0 & $1(8)$ & $3(14)$ \\
\hline Unknown & 0 & $1(33)$ & 0 & $1(5)$ \\
\hline
\end{tabular}

ECOG PS Eastern Cooperative Oncology Group performance status, Gem gemcitabine, NSCLC non-small-cell lung cancer, Paz pazopanib 
Table 2 Summary of exposure and dose-limiting toxicity

\begin{tabular}{|c|c|c|c|c|}
\hline \multirow[t]{2}{*}{ Dose level } & \multirow[t]{2}{*}{$\begin{array}{l}\text { Number } \\
\text { of patients }\end{array}$} & \multirow[t]{2}{*}{$\begin{array}{l}\text { Number } \\
\text { of DLTs }{ }^{\mathrm{a}}\end{array}$} & \multicolumn{2}{|c|}{$\begin{array}{l}\text { Median number } \\
\text { of cycles (range) }\end{array}$} \\
\hline & & & $\mathrm{Paz}$ & Gem \\
\hline Paz400/Gem1000 & 6 & $1^{\mathrm{b}}$ & $5.5(2-17)$ & $5.5(2-17)$ \\
\hline Paz800/Gem1000 & 3 & 0 & $6(5-12)$ & $6(5-12)$ \\
\hline Paz800/Gem1250 & 13 & $1^{\mathrm{c}}$ & $4(1-14)$ & $4(1-14)$ \\
\hline
\end{tabular}

DLT dose-limiting toxicity, Gem gemcitabine, Paz pazopanib

a Observed during Cycles 1 and 2 during the dose-escalation phase

b Grade 4 thrombocytopenia

c Grade 3 fatigue

\section{Exposure}

The median number of cycles of pazopanib and gemcitabine received in the Paz400/Gem1000 $(n=6)$, Paz800/ Gem1000 $(n=3)$, and Paz800/Gem1250 $(n=13)$ dose levels were 5.5 cycles (range 2-17), 6 cycles (range 5-12), and 4 cycles (range 1-14), respectively. The median dose intensity for each of the three dose levels tested was as follows: Paz400/Gem1000, $99 \% / 78 \%$; Paz800/Gem1000, $95 \% / 78 \%$; and Paz800/Gem1250, $100 \% / 81 \%$. At least one pazopanib dose delay was observed in 12 patients (55\%), and 17 patients (77 \%) had at least one gemcitabine dose delay. Across all dose levels, the most common AEs leading to a dose delay were neutropenia (three patients, $14 \%$ ), thrombocytopenia ( 2 patients, $9 \%$ ), and diarrhea (two patients, $9 \%$ ). Two patients $(9 \%)$ required at least one pazopanib dose reduction, whereas 12 patients $(55 \%)$ required at least one gemcitabine dose reduction. The most common AEs resulting in dose reductions were neutropenia (41\%), thrombocytopenia (14\%), and hypertension (5\%).

Safety and tolerability

The most common treatment-related AEs reported in patients across all dose levels were fatigue (68\%), neutropenia $(59 \%)$, nausea $(55 \%)$, and decreased appetite (50\%; Table 3). The majority of treatment-related AEs were Grade 1 or 2 . Seven patients $(32 \%)$ across all dose levels experienced Grade 4 treatment-related AEs of neutropenia and thrombocytopenia. The majority of patients (41\%) discontinued treatment due to disease progression; three patients $(14 \%)$ discontinued due to AEs (Grade 3 increased alanine aminotransferase, Grade 1 hematoma, and Grade 3 fatigue), two patients (9\%) discontinued at the investigator's discretion, and four patients $(18 \%)$ withdrew consent. One treatment-related death (Grade 5 pneumonia) was reported in Dose Level 1 (Paz400/ Gem1000).

\section{Pharmacokinetics}

There was considerable variability in plasma concentrations of pazopanib, gemcitabine, and $\mathrm{dFdU}$ during the sparse sampling for patients in the dose-escalation phase (Table 4). Given this variability and the relatively small number of patients enrolled in each cohort, dose

Table 3 Treatment-related adverse events occurring in $\geq 10 \%$ of overall patient population

\begin{tabular}{|c|c|c|c|c|}
\hline Adverse event $(n \%)$ & Paz400/Gem1000 $(n=6)$ & Paz800/Gem1000 $(n=3)$ & Paz800/Gem1250 $(n=13)$ & Total $(N=22)$ \\
\hline Fatigue & $4(67)$ & $3(100)$ & $8(62)$ & $15(68)$ \\
\hline Neutropenia & $4(67)$ & $1(33)$ & $8(62)$ & $13(59)$ \\
\hline Nausea & $4(67)$ & $2(67)$ & $6(46)$ & $12(55)$ \\
\hline Decreased appetite & $2(33)$ & $1(33)$ & $8(62)$ & $11(50)$ \\
\hline Leukopenia & $2(33)$ & $1(33)$ & $7(54)$ & $10(45)$ \\
\hline Thrombocytopenia & $3(50)$ & $1(33)$ & $5(38)$ & $9(41)$ \\
\hline Diarrhea & $3(50)$ & $1(33)$ & $4(31)$ & $8(36)$ \\
\hline Vomiting & $1(17)$ & $1(33)$ & $4(31)$ & $6(27)$ \\
\hline ALT increased & $1(17)$ & $2(67)$ & $3(23)$ & $6(27)$ \\
\hline Dysgeusia & $2(33)$ & $3(100)$ & $1(8)$ & $6(27)$ \\
\hline Stomatitis & $4(67)$ & $1(33)$ & 0 & $5(23)$ \\
\hline Alopecia & 0 & 0 & $4(31)$ & $4(18)$ \\
\hline Hair color changes & $1(17)$ & 0 & $3(23)$ & $4(18)$ \\
\hline AST increased & $1(17)$ & $1(33)$ & $2(15)$ & $4(18)$ \\
\hline Epistaxis & 0 & $1(33)$ & $3(23)$ & $4(18)$ \\
\hline Dry skin & $1(17)$ & $1(33)$ & $1(8)$ & $3(14)$ \\
\hline
\end{tabular}

$A L T$ alanine aminotransferase, $A S T$ aspartate aminotransferase, Gem gemcitabine, Paz pazopanib 
Table 4 Summary of concentrations of pazopanib, gemcitabine, and dFdU from sparse sampling in the dose-escalation phase

\begin{tabular}{|c|c|c|c|c|}
\hline Dose cohort & Cycle day & Nominal time & $N$ & Median concentration (range) \\
\hline \multicolumn{5}{|c|}{ Pazopanib concentrations ( $\mu \mathrm{g} / \mathrm{mL})$} \\
\hline \multirow[t]{3}{*}{ Paz400/Gem 1000} & C1D1 & $3.5 \mathrm{~h}$ & 6 & $22.5(6.5-42.8)$ \\
\hline & C1D8 & Pre-dose & 6 & $22.3(10.4-37.1)$ \\
\hline & $\mathrm{C} 2 \mathrm{D} 1$ & Pre-dose & 6 & $21.6(1.0-30.8)$ \\
\hline \multirow[t]{3}{*}{ Paz800/Gem1000 } & C1D1 & $3.5 \mathrm{~h}$ & 3 & $51.0(36.0-52.1)$ \\
\hline & C1D8 & Pre-dose & 3 & $24.9(11.9-52.4)$ \\
\hline & $\mathrm{C} 2 \mathrm{D} 1$ & Pre-dose & 3 & $22.9(10.9-80.3)$ \\
\hline \multirow[t]{3}{*}{ Paz800/Gem1250 } & C1D1 & $3.5 \mathrm{~h}$ & 7 & $20.7(7.6-43.3)$ \\
\hline & C1D8 & Pre-dose & 6 & $23.9(8.6-37.6)$ \\
\hline & $\mathrm{C} 2 \mathrm{D} 1$ & Pre-dose & 4 & $19.1(7.4-25.1)$ \\
\hline \multicolumn{5}{|c|}{ Gemcitabine concentrations $(\mathrm{ng} / \mathrm{mL})^{\mathrm{a}}$} \\
\hline \multirow[t]{2}{*}{ Paz400/Gem1000 } & C1D1 & $0.5 \mathrm{~h}$ & 6 & $10,532(1,318-11,647)$ \\
\hline & C1D8 & $0.5 \mathrm{~h}$ & 6 & $9,691(994-25,529)$ \\
\hline \multirow[t]{2}{*}{ Paz800/Gem1000 } & C1D1 & $0.5 \mathrm{~h}$ & 3 & $10,820(9,375-13,881)$ \\
\hline & C1D8 & $0.5 \mathrm{~h}$ & 3 & $18,936(7,913-22,638)$ \\
\hline \multirow[t]{2}{*}{ Paz800/Gem1250 } & C1D1 & $0.5 \mathrm{~h}$ & 7 & $17,854(8,130-22,541)$ \\
\hline & C1D8 & $0.5 \mathrm{~h}$ & 6 & $16,006(5,274-21,032)$ \\
\hline \multicolumn{5}{|c|}{$\mathrm{dFdU}$ concentrations (ng/mL) } \\
\hline \multirow[t]{3}{*}{ Paz400/Gem1000 } & C1D1 & $0.5 \mathrm{~h}$ & 6 & $29,672(15,654-54,539)$ \\
\hline & C1D8 & Pre-dose & 6 & $301(0-997)$ \\
\hline & C1D8 & $0.5 \mathrm{~h}$ & 6 & $31,005(17,927-38,586)$ \\
\hline \multirow[t]{3}{*}{ Paz800/Gem1000 } & C1D1 & $0.5 \mathrm{~h}$ & 3 & $41,032(22,844-43,207)$ \\
\hline & C1D8 & Pre-dose & 3 & $743(686-1,745)$ \\
\hline & C1D8 & $0.5 \mathrm{~h}$ & 3 & $40,789(24,614-41,044)$ \\
\hline \multirow[t]{3}{*}{ Paz800/Gem1250 } & C1D1 & $0.5 \mathrm{~h}$ & 7 & $34,175(21,999-45,346)$ \\
\hline & C1D8 & Pre-dose & 6 & $889(0-1,678)$ \\
\hline & C1D8 & $0.5 \mathrm{~h}$ & 6 & $35,378(27,767-49,545)$ \\
\hline
\end{tabular}

$C$ cycle, $D$ day, $d F d U 2^{\prime}, 2^{\prime}$-difluorodeoxyuridine, Gem gemcitabine, $h$ hour, Paz pazopanib

${ }^{\text {a }}$ C1D8 pre-dose concentrations for gemcitabine were all less than lower limit of quantitation $(50 \mathrm{ng} / \mathrm{mL})$

proportionality could not be reasonably assessed. In addition, elevations in plasma levels of pazopanib, gemcitabine, or dFdU were not associated with occurrence of either of the 2 DLTs noted above (i.e., Grade 4 thrombocytopenia reported on Cycle 1 Day 8 for one patient in the Paz400/ Gem1000 group and Grade 3 fatigue on Cycle 1 Day 1 for one patient in the Paz800/Gem1250 cohort).

Six patients in the cohort-expansion phase had frequent sampling performed for pharmacokinetic analysis. Median concentration-time profiles for pazopanib (Cycle 2 Day 1) and for gemcitabine and $\mathrm{dFdU}$ after dosing of gemcitabine alone (Cycle 1 Day 1) and in combination with pazopanib (Cycle 2 Day 1) are presented in Fig. 1. The median gemcitabine and $\mathrm{dFdU}$ concentration profiles from gemcitabine alone and from gemcitabine after 21 days of pazopanib administration appear very similar. Gemcitabine is rapidly transformed into $\mathrm{dFdU}$ which quickly achieves appreciably greater concentrations with a much longer elimination half-life. Summary pharmacokinetic parameters for pazopanib, gemcitabine, and $\mathrm{dFdU}$ are presented in Table 5, along with statistical results from the ANOVA investigating the effect of pazopanib on gemcitabine pharmacokinetics. Because of missed samples, not all pharmacokinetic parameters could be estimated for all patients. Results from the statistical analysis suggest that gemcitabine and dFdU systemic exposures are slightly higher with pazopanib co-administration, with $C_{\max }$ and AUC geometric least squares mean ratios of 1.06 (90\% CI: $0.62,1.84)$ and 1.26 (90\% CI: $0.79,2.01)$, respectively, for gemcitabine, and ratios of 0.95 (90\% CI: $0.90,1.00)$ and 1.21 (90 \% CI: 1.08, 1.36), respectively, for dFdU. This overall assessment is limited by both pharmacokinetic variability and small sample size.

\section{Clinical activity}

One partial objective response was initially reported on Day 42 (end of Cycle 2) and sustained through the last 
Fig. 1 Median pazopanib, gemcitabine, and $\mathrm{dFdU}$ concentration-time profiles from patients in the cohortexpansion phase: gemcitabine $\left(1,250 \mathrm{mg} / \mathrm{m}^{2}\right.$ by $30-\mathrm{min}$ infusion) was administered alone on Cycle 1 Day 1 (C1D1) and in combination with oral pazopanib $(800 \mathrm{mg})$ on Cycle 2 Day 1 (C2D1); pazopanib was administered once daily beginning on C1D2, and gemcitabine was administered on Days 1 and 8 of the 21-day cycle

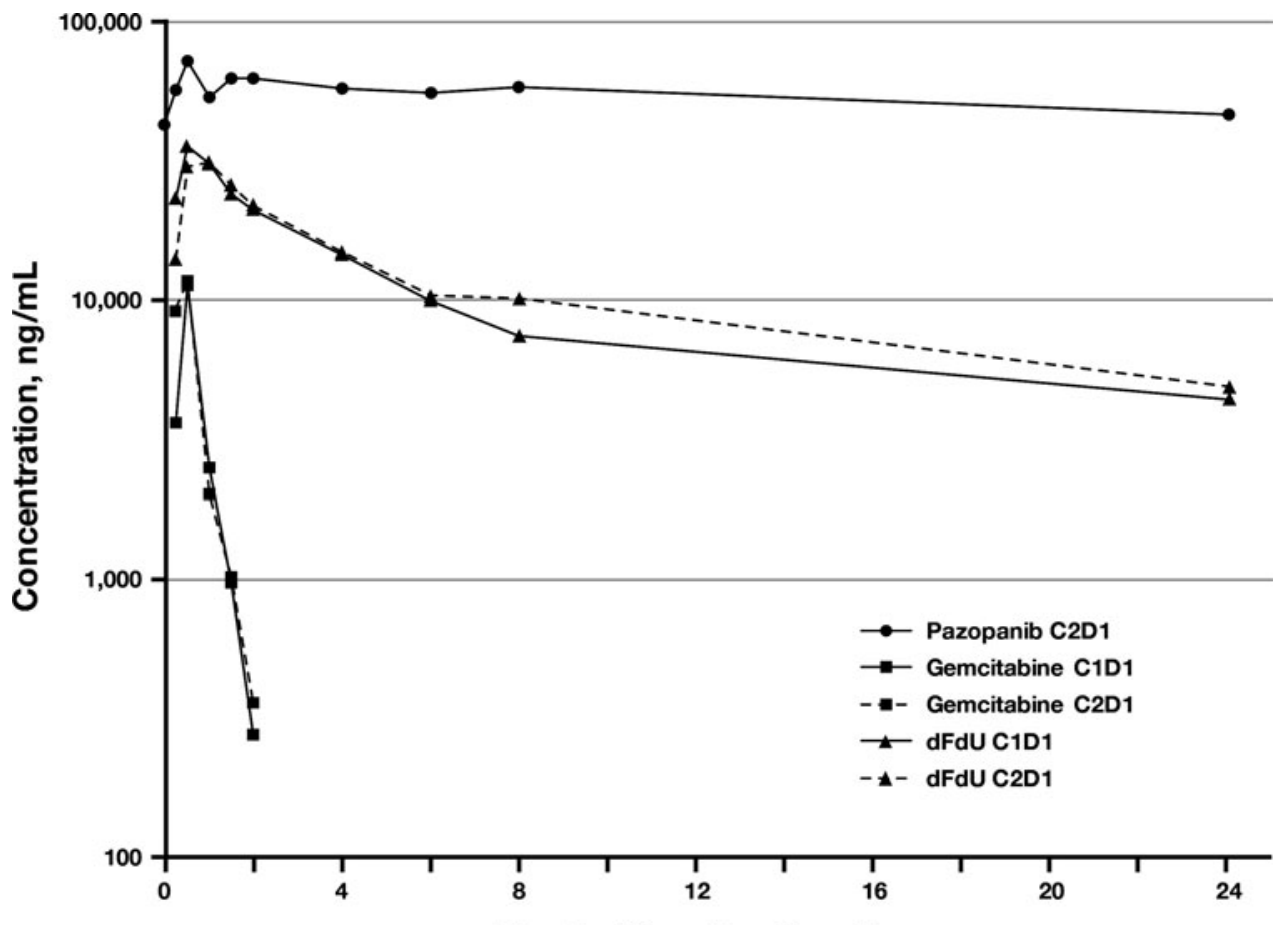

Nominal Sampling Time, hours

Table 5 Summary of pharmacokinetic parameter estimates and statistical results for pazopanib, gemcitabine, and dFdU from the cohortexpansion phase

\begin{tabular}{|c|c|c|c|c|c|c|c|}
\hline \multirow[t]{2}{*}{ PK Parameter } & \multirow{2}{*}{$\begin{array}{l}\text { Pazopanib } \\
\text { C2D1 }\end{array}$} & \multicolumn{3}{|l|}{ Gemcitabine } & \multicolumn{3}{|l|}{$\mathrm{dFdU}$} \\
\hline & & $\mathrm{C} 1 \mathrm{D} 1^{\mathrm{a}}$ & $\mathrm{C} 2 \mathrm{D} 1^{\mathrm{a}}$ & $\begin{array}{l}\text { GLM Ratio } \\
(90 \% \text { CI })^{\mathrm{b}}\end{array}$ & $\mathrm{C} 1 \mathrm{D} 1^{\mathrm{a}}$ & $\mathrm{C} 2 \mathrm{D} 1^{\mathrm{a}}$ & $\begin{array}{l}\text { GLM Ratio } \\
(90 \% \text { CI })^{b}\end{array}$ \\
\hline \multirow[t]{2}{*}{$C_{\max }^{\mathrm{c}}$} & $73.3(5)$ & $17,380(4)$ & $18,802(4)$ & 1.06 & $36,134(6)$ & $33,911(5)$ & 0.95 \\
\hline & $31.8-98.8$ & $10,623-28,664$ & $9,175-33,898$ & $(0.62,1.84)$ & $32,129-44,210$ & $28,491-39,942$ & $(0.90,1.00)$ \\
\hline \multirow[t]{2}{*}{$T_{\max }(\mathrm{h})$} & $2(5)$ & $0.50(4)$ & $0.52(4)$ & - & $0.67(6)$ & $1.13(5)$ & - \\
\hline & $1.58-9$ & $0.32-0.53$ & $0.25-0.57$ & & $0.50-1.02$ & $0.57-1.75$ & \\
\hline \multirow[t]{2}{*}{$\operatorname{AUC}_{(0-24)}\left(h^{*} \mu \mathrm{g} / \mathrm{mL}\right)$} & $1,340(5)$ & - & - & - & - & - & - \\
\hline & $680-1,777$ & & & & & & \\
\hline \multirow[t]{2}{*}{$\operatorname{AUC}_{(0-\infty)}\left(h^{*} \mathrm{ng} / \mathrm{mL}\right)$} & - & 9,953 (4) & $11,306(4)$ & 1.26 & $333,145(6)$ & $350,950(5)$ & 1.21 \\
\hline & & $6,522-19,678$ & $7,025-21,471$ & $(0.79,2.01)$ & $244,765-393,908$ & $306,839-547,673$ & $(1.08,1.36)$ \\
\hline \multirow[t]{2}{*}{$t^{1 / 2}(\mathrm{~h})$} & $47(4)$ & $0.26(6)$ & $0.34(5)$ & 1.28 & $11.9(6)$ & $12.3(5)$ & 1.06 \\
\hline & $32.1-69.5$ & $0.26-0.31$ & $0.31-0.44$ & $(1.16,1.41)$ & $10.9-25.7$ & $11.4-23.7$ & $(0.91,1.24)$ \\
\hline \multirow[t]{2}{*}{$\mathrm{CL}\left(\mathrm{L} / \mathrm{h} / \mathrm{m}^{2}\right)$} & - & $135(4)$ & $117(4)$ & 0.79 & - & - & - \\
\hline & & $64-192$ & $58-178$ & $(0.50,1.27)$ & & & \\
\hline \multirow[t]{2}{*}{$C_{\min }(\mu \mathrm{g} / \mathrm{mL})$} & $37.5(5)$ & - & - & - & - & - & - \\
\hline & $21.5-56.8$ & & & & & & \\
\hline
\end{tabular}

$A U C_{(0-24)}$ area under the concentration-time curve $0-24 \mathrm{~h}, A U C_{(0-\infty)}$ AUC extrapolated to infinity, $C$ cycle, $C I$ confidence interval, $C L$ clearance, $C_{\max }$ maximum concentration, $C_{\min }$ minimum concentration, $D$ day, $d F d U 2^{\prime}, 2^{\prime}$-difluorodeoxyuridine, $G L M$ geometric least squares mean, $h$ hour, $P K$ pharmacokinetic, $T_{\max }$ time of $C_{\max }, t_{1 / 2}$ elimination half-life

${ }^{a}$ Values denote median (n) and range

b Values denote GLM ratio of (C2D1/C1D1) and $90 \%$ CI from analysis of variance

${ }^{c}$ Units for $C_{\max }$ are $\mu \mathrm{g} / \mathrm{mL}$ for pazopanib and $\mathrm{ng} / \mathrm{mL}$ for gemcitabine and $\mathrm{dFdU}$ 
assessment on Day 327 in a female patient with melanoma in the Paz800/Gem1250 cohort. Fourteen patients had stable disease at 1 or more disease assessment time points; three of these patients had stable disease for at least 12 cycles (cholangiocarcinoma, 17 cycles; melanoma, 14 cycles; and colorectal cancer, 12 cycles).

\section{Discussion}

This study demonstrated that pazopanib and gemcitabine can be safely administered at doses similar to those given as monotherapy. The most common AEs experienced by patients receiving the combination of pazopanib and gemcitabine were consistent with the known safety profile of each agent individually. The most frequently reported treatment-related AEs were fatigue, neutropenia, nausea, decreased appetite, and thrombocytopenia.

No apparent pharmacokinetic interaction between pazopanib and gemcitabine was observed. However, the assessment was limited by extensive interpatient variability and small sample size. Although this study did not have a period of pazopanib monotherapy without gemcitabine, the pazopanib pharmacokinetic parameters were similar to historical results from pazopanib $800 \mathrm{mg}$ monotherapy [19], suggesting no apparent effect of gemcitabine on pazopanib pharmacokinetics. The pharmacokinetic parameters for gemcitabine and $\mathrm{dFdU}$ from both gemcitabine alone (Cycle 1 Day 1) and in combination with pazopanib (Cycle 2 Day 1) were also similar to historical estimates [20, 21].

Preliminary clinical activity was characterized by one patient with a partial objective response and 14 patients with stable disease, including three patients in whom the duration of stable disease ranged from 12 to 17 treatment cycles (21-day cycles).

In summary, therapeutic doses associated with efficacy of both pazopanib and gemcitabine monotherapy were achieved. There was no apparent pharmacokinetic interaction at the highest dose level tested (Paz800/Gem1250), although interindividual variability and small sample size limit the robustness of this inference. The combination of pazopanib and gemcitabine was generally well tolerated, and Phase 2 studies of this combination are warranted.

\begin{abstract}
Acknowledgments The authors wish to thank the patients who participated in this study and the study coordinators and all clinical research assistants for their contributions to the conduct of this study. Editorial assistance was provided by Jerome F. Sah, PhD, ProEd Communications Inc., Beachwood, Ohio, and supported by GlaxoSmithKline. This study (NCT00678977; VEG109599) was supported by GlaxoSmithKline Pharmaceuticals, Philadelphia, PA.
\end{abstract}

Conflict of interest S. Rubin, S. Weller, and D. M. Gibson are employed by GlaxoSmithKline and hold company stock; H. A. Ball was employed by GlaxoSmithKline and holds company stock. J. Botbyl is employed by Provonix, which has a contractual relationship with GlaxoSmithKline. R. Plummer and M. Scheulen have received funding from GlaxoSmithKline for clinical trial costs. The other authors disclosed no potential conflicts of interest. The authors have full control of all primary data and agree to allow the journal to review their data if requested.

Open Access This article is distributed under the terms of the Creative Commons Attribution License which permits any use, distribution, and reproduction in any medium, provided the original author(s) and the source are credited.

\section{References}

1. Sternberg CN, Davis ID, Mardiak J et al (2010) Pazopanib in locally advanced or metastatic renal cell carcinoma: results of a randomized phase III trial. J Clin Oncol 28: $1061-1068$

2. van der Graaf WT, Blay JY, Chawla SP et al (2012) Pazopanib for metastatic soft-tissue sarcoma (PALETTE): a randomised, double-blind, placebo-controlled phase 3 trial. Lancet 379: $1879-1886$

3. Altorki N, Lane ME, Bauer T et al (2010) Phase II proof-ofconcept study of pazopanib monotherapy in treatment-naive patients with stage I/II resectable non-small-cell lung cancer. J Clin Oncol 28:3131-3137

4. Bible KC, Suman VJ, Molina JR et al (2010) Efficacy of pazopanib in progressive, radioiodine-refractory, metastatic differentiated thyroid cancers: results of a phase 2 consortium study. Lancet Oncol 11:962-972

5. Friedlander M, Hancock KC, Rischin D, Messing MJ, Stringer CA, Matthys GM, Ma B, Hodge JP, Lager JJ (2010) A Phase II, open-label study evaluating pazopanib in patients with recurrent ovarian cancer. Gynecol Oncol 119:32-37

6. Sleijfer S, Papai Z, Le Cesne A et al (2007) Phase II study of pazopanib (GW786034) in patients (pts) with relapsed or refractory soft tissue sarcoma (STS): EORTC 62043 (abstr 10031). J Clin Oncol 18S:552s

7. Monk BJ, Mas Lopez L, Zarba JJ et al (2010) Phase II, open-label study of pazopanib or lapatinib monotherapy compared with pazopanib plus lapatinib combination therapy in patients with advanced and recurrent cervical cancer. J Clin Oncol 28: 3562-3569

8. Burris HA 3rd, Moore MJ, Andersen J et al (1997) Improvements in survival and clinical benefit with gemcitabine as first-line therapy for patients with advanced pancreas cancer: a randomized trial. J Clin Oncol 15:2403-2413

9. Carmichael J, Possinger K, Phillip P, Beykirch M, Kerr H, Walling J, Harris AL (1995) Advanced breast cancer: a phase II trial with gemcitabine. J Clin Oncol 13:2731-2736

10. Lund B, Hansen OP, Neijt JP, Theilade K, Hansen M (1995) Phase II study of gemcitabine in previously platinum-treated ovarian cancer patients. Anticancer Drugs 6:61-62

11. Sandler AB, Nemunaitis J, Denham C et al (2000) Phase III trial of gemcitabine plus cisplatin versus cisplatin alone in patients with locally advanced or metastatic non-small-cell lung cancer. J Clin Oncol 18:122-130

12. Maki RG, Wathen JK, Patel SR et al (2007) Randomized phase II study of gemcitabine and docetaxel compared with gemcitabine alone in patients with metastatic soft tissue sarcomas: results of sarcoma alliance for research through collaboration study 002 [corrected]. J Clin Oncol 25:2755-2763 
13. Abbruzzese JL, Grunewald R, Weeks EA et al (1991) A phase I clinical, plasma, and cellular pharmacology study of gemcitabine. J Clin Oncol 9:491-498

14. Martin C, Pollera CF (1996) Gemcitabine: safety profile unaffected by starting dose. Int J Clin Pharmacol Res 16:9-18

15. Gaitskell K, Martinek I, Bryant A, Kehoe S, Nicum S, Morrison J (2011) Angiogenesis inhibitors for the treatment of ovarian cancer. Cochrane Database Syst Rev 9:CD007930

16. Ulahannan SV, Brahmer JR (2011) Antiangiogenic agents in combination with chemotherapy in patients with advanced nonsmall cell lung cancer. Cancer Invest 29:325-337

17. Wagner AD, Arnold D, Grothey AA, Haerting J, Unverzagt S (2009) Anti-angiogenic therapies for metastatic colorectal cancer. Cochrane Database Syst Rev 3:CD005392

18. Therasse P, Arbuck SG, Eisenhauer EA et al (2000) New guidelines to evaluate the response to treatment in solid tumors.
European Organization for Research and Treatment of Cancer, National Cancer Institute of the United States, National Cancer Institute of Canada. J Natl Cancer Inst 92:205-216

19. Goh BC, Reddy NJ, Dandamudi UB et al (2010) An evaluation of the drug interaction potential of pazopanib, an oral vascular endothelial growth factor receptor tyrosine kinase inhibitor, using a modified Cooperstown $5+1$ cocktail in patients with advanced solid tumors. Clin Pharmacol Ther 88:652-659

20. Eli Lilly and Company (2012) Gemzar (gemcitabine for injection) powder prescribing information. Revised February 2011 [cited January 5, 2012]. Available from: http://pi.lilly.com/us/ gemzar.pdf

21. Jiang X, Galettis P, Links M, Mitchell PL, McLachlan AJ (2008) Population pharmacokinetics of gemcitabine and its metabolite in patients with cancer: effect of oxaliplatin and infusion rate. $\mathrm{Br} \mathrm{J}$ Clin Pharmacol 65:326-333 